\title{
Analysis of Multi-Agent-Based Adaptive Droop-Controlled AC Microgrids with PSCAD: Modeling and Simulation
}

\author{
Zhongwen $\mathrm{Li}^{*}{ }^{* * *}$, Chuanzhi Zang ${ }^{*}$, Peng Zeng ${ }^{*}$, Haibin $\mathrm{Yu}^{\dagger}$, Hepeng $\mathrm{Li}^{*}$, and Shuhui $\mathrm{Li}^{* * *}$ \\ ${ }^{* \dagger}$ Lab. of Networked Control Systems, Shenyang Institute of Automation, Chinese Academy of Sciences, Shenyang, China \\ ${ }^{* *}$ University of Chinese Academy of Sciences, Beijing, China \\ ${ }^{* * *}$ Department of Electrical and Computer Engineering, The University of Alabama, Tuscaloosa, AL, USA
}

\begin{abstract}
A microgrid (MG) with integrated renewable energy resources can benefit both utility companies and customers. As a result, they are attracting a great deal of attention. The control of a MG is very important for the stable operation of a MG. The droop-control method is popular since it avoids circulating currents among the converters without using any critical communication between them. Traditional droop control methods have the drawback of an inherent trade-off between power sharing and voltage and frequency regulation. An adaptive droop control method is proposed, which can operate in both the island mode and the grid-connected mode. It can also ensure smooth switching between these two modes. Furthermore, the voltage and frequency of a MG can be restored by using the proposed droop controller. Meanwhile, the active power can be dispatched appropriately in both operating modes based on the capacity or running cost of the Distributed Generators (DGs). The global information (such as the average voltage and output active power of the MG and so on) required by the proposed droop control method to restore the voltage and frequency deviations can be acquired distributedly based on the Multi Agent System (MAS). Simulation studies in PSCAD demonstrate the effectiveness of the proposed control method.
\end{abstract}

Key words: Distributed Generator (DG), Droop control, Frequency restoration, Global information discovery, Microgrid, Multi-agent system,

\section{INTRODUCTION}

In order to meet the increasing demand for electricity, Distributed Generation (DG) based on Renewable Energy Sources (RES) (such as photovoltaic, fuel cells and wind-power) and storage systems is emerging as a promising new technology [1]. By using Distributed Generators (DGs), the distance between the generation and the loads is reduced, which can reduce losses, and postpones investments in new transmission and large scale generation systems [2]. Microgrids

Manuscript received Jul. 23, 2014; accepted Dec. 31, 2014

Recommended for publication by Associate Editor Jae-Do Park.

'Corresponding Author: yhb@sia.cn

Tel: +86-024-2397-0118, Fax: +86-024-2397-0043, Shenyang Institute of Automation, Chinese Academy of Sciences

*University of Chinese Academy of Sciences, Beijing, China

${ }^{* *}$ Lab. of Networked Control Systems, Shenyang Institute of Automation, Chinese Academy of Sciences, Shenyang, China

${ }^{* * *}$ Department of Electrical and Computer Engineering, The University of Alabama, USA
(MGs) are attracting a great deal of attention since they integrate these DGs into the main grid reliably and cleanly [1], [3]-[5]. For utility companies, MGs can operate as a single dispatchable unit to provide power or ancillary services. For customers, they can improve power quality by supporting local voltage and frequencies [6], [7].

The droop control methods are presented as wireless control techniques that avoid circulating currents among the converters without using any critical communications between them. A lot of studies suggest that droop control methods are the best option for controlling the DGs in MGs [3], [8]-[10]. Furthermore, droop-control methods are often applied in many experimental MGs because of the advantages they offer, such as flexibility and absence of critical communications [1], [11].

For droop control methods, an inherent trade-off between power sharing and voltage and frequency regulation is one of its drawbacks. This is due to the fact that if the droop coefficients are increased, then good power sharing is achieved 


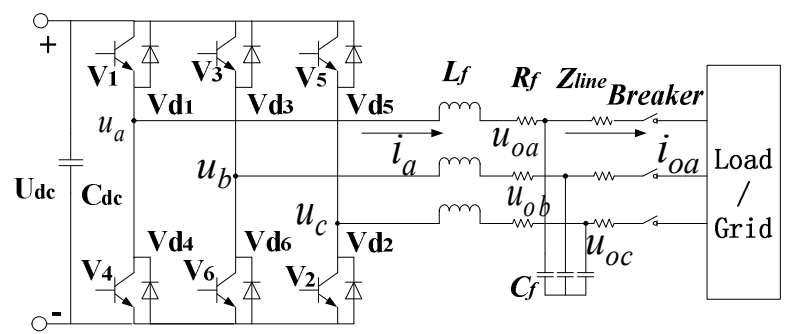

Fig. 1. Circuit structure of three-phase VSI.

at the expense of degrading the voltage/frequency regulation. On the other hand, if the droop coefficients are decreased, then good voltage/frequency regulation is achieved at the expense of degrading the power sharing [12]. Nowadays, many researchers are focusing on improving droop control and many improved methods have been proposed. A modified droop controller was discussed in [13]-[18], but the frequency and voltage amplitude experience deviations in the steady state. A restoration control was proposed in [19], [20], but it must be carried out by means of a central controller, which requires some form of communication between the restoration control and the DGs, which is complex and not reliable for a single point of failure.

In order to restore the MG voltage magnitude and frequency while achieving accurate power sharing, centralized active load sharing techniques and average load sharing techniques have been proposed in [20] and [21], respectively. However, these techniques reduce system reliability and expandability due to their dependency on critical intercommunication lines among modules [22].

The Multi Agent System (MAS) is one of the most popular distributed control strategies. The convergence, robustness and stability analysis of the multi agent based algorithm was discussed in [23]-[25], which is helpful for application to MGs. In recent years, several global information discovery algorithms based on MAS have been proposed and applied to MGs [25]-[27].

In this paper, an adaptive droop control method is proposed, which can operate in both the island mode and the grid-connected mode. In addition, it can shift smoothly between these two modes. Furthermore, the voltage and frequency of a MG can be restored. Meanwhile, the active power can be dispatched appropriately in both of the operating modes based on the capacity or the running cost of the DGs. Based on the MAS, the global information (which is required by the proposed droop control method to restore the voltage and frequency deviation ) can be acquire distributedly.

The global information discovered in this paper includes the status information (such as the state of each of the DGs and the weight factor to the dispatch active load) and the performance metric (such as the average voltage and the output active power of the MG). During the information discovery process, the agents only need to communicate with their corresponding neighbors. Furthermore, the global information discovery algorithm is independent of the system configuration. Thus, it can be applied to MG systems with any structure, such as radial, mesh and mixed.

The main novel contributions of this paper are:

1) A MAS based adaptive droop control method for microgrids was proposed and the stability of this control architecture was analyzed through a small-signal model.

2) A global information discovery algorithm based on the MAS is applied for the DGs to discovery global information distributedly. In addition, the frequency and voltage deviation can be restored with the adaptive droop controller using the acquired global information, which overcomes the drawback of traditional droop control methods.

3) The transient and steady state performances of the adaptive droop-controlled $\mathrm{MG}$ are verified by simulations. It is shown to have better performance than traditional droop control methods.

This paper is organized as follows. In section II, both the MG system model and the control method are presented. Section III proposes the MAS-based global information discovery algorithm. Section IV presents simulations and results. Finally, Section V concludes this paper.

\section{MICROGRID SYSTEM MODEL AND CONTROL METHOD}

\section{A. Voltage Source Inverter Model}

In MGs, Voltage Source Inverters (VSIs) are very popular because they can provide ride-through capability and power-quality enhancement to DGs [20]. These inverters are required to operate in both the grid-connected mode and the island mode, and they often change their behavior from voltage to current sources in the grid-connected mode. In order to operate in both the grid-connected and islanded modes, VSIs are required to control the exported or imported power to the main grid to stabilize the MG [28]. The circuit structure of a three-phase VSI is shown in Fig. 1.

In Fig. $1, L_{f}$ is the inductance of the LC filter, $R_{f}$ is the resistance of the inductor, $C_{f}$ is the capacitance of the LC filter, $u_{a}, u_{b}, u_{c}$ are the three phase output voltages of the inverter, $u_{o a}, u_{o b}, u_{o c}$ are the voltages of the capacitor, $Z_{\text {line }}$ is the impedance of the transmission-line, $i_{a}$ is the current of the filter inductor, and $i_{o a}$ is the current in the transmission-line.

By using a $d-q$ transformation, the three-phase voltage and current can be transformed to the $d-q$ rotating reference frame. Then the active and reactive powers can be decoupled by using a $d-q$ transformation [17]. Details of the $a b c / d q$ transformation and its sign convention are shown in Eq. (1), 

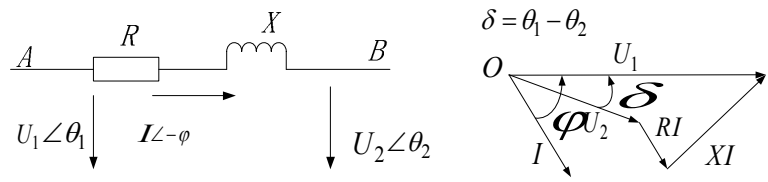

Fig. 2. Power flow and vector diagram between the main grid and the inverter interfaced DG.

and the $d q / a b c$ transformation is shown in Eq. (2).

$$
\begin{gathered}
C_{a b c / d q}=\frac{2}{3} \cdot\left[\begin{array}{ccc}
\cos (w t) & \cos (w t-2 \pi / 3) & \cos (w t+2 \pi / 3) \\
-\sin (w t) & -\sin (w t-2 \pi / 3) & -\sin (w t+2 \pi / 3)
\end{array}\right] \\
C_{d q / a b c}=\left[\begin{array}{cc}
\cos (w t) & -\sin (w t) \\
\cos (w t-2 \pi / 3) & -\sin (w t-2 \pi / 3) \\
\cos (w t+2 \pi / 3) & -\sin (w t+2 \pi / 3)
\end{array}\right]
\end{gathered}
$$

By using the former $d-q$ transformation, the mathematical equation of the inverter circuit can be obtained from Fig. 1 as Eqns. (3)-(6). In this paper, the subscripts $d$ and $q$ represent the $d$-axis component and the $q$-axis component of some variable after the $d-q$ transformation.

$$
\begin{gathered}
L_{f} \cdot \dot{i}_{d}=u_{d}-u_{o d}-R_{f} \cdot i_{d}+w \cdot L_{f} \cdot i_{q} \\
L_{f} \cdot \dot{i}_{q}=u_{q}-u_{o q}-R_{f} \cdot i_{q}-w \cdot L_{f} \cdot i_{d} \\
C_{f} \cdot \dot{u}_{o d}=i_{d}-i_{o d}+w \cdot C_{f} \cdot u_{o q} \\
C_{f} \cdot \dot{u}_{o q}=i_{q}-i_{o q}-w \cdot C_{f} \cdot u_{o d}
\end{gathered}
$$

Eqns. (3)-(4) demonstrate that $i_{d}, i_{q}$ are not only affected by $u_{o d}, u_{o q}$, but that they are affected by the coupling currents $w \cdot L_{f} \cdot i_{q}$ and $w \cdot L_{f} \cdot i_{d}$. Reference [29] shows that the feedforward quantities $u_{o d}, u_{o q}$ and cross decoupled quantities $w \cdot L_{f} \cdot i_{q}$ and $w \cdot L_{f} \cdot i_{d}$ are used to achieve independent current control in the $d-q$ axis. Thus, the inner current control loop is designed as Eqns. (7)-(8) [29] to eliminate the current coupling.

$$
\begin{aligned}
& u_{d}=\left(K_{p i}+\frac{K_{I i}}{s}\right) \cdot\left(i_{d}^{*}-i_{d}\right)-w \cdot L_{f} \cdot i_{q}+u_{o d} \\
& u_{q}=\left(K_{p i}+\frac{K_{I i}}{s}\right) \cdot\left(i_{q}^{*}-i_{q}\right)+w \cdot L_{f} \cdot i_{d}+u_{o q}
\end{aligned}
$$

Applying Eqns. (7)-(8) into Eqns. (3)-(4) the following are obtained:

$$
\begin{aligned}
& \left(s \cdot L_{f}+R_{f}\right) i_{d}=\left(K_{p i}+\frac{K_{I i}}{s}\right) \cdot\left(i_{d}^{*}-i_{d}\right) \\
& \left(s \cdot L_{f}+R_{f}\right) i_{q}=\left(K_{p i}+\frac{K_{I i}}{s}\right) \cdot\left(i_{q}^{*}-i_{q}\right)
\end{aligned}
$$

As a result, $i_{d}$ and $i_{q}$ can be controlled separately without coupling.
Eqns. (5)-(6) indicate that $u_{o d}, u_{o q}$ are affected by $i_{o d}, i_{o q}$ and the coupling voltages $w \cdot C_{f} \cdot u_{o q}$ and $w \cdot C_{f} \cdot u_{o d}$. Reference [29] indicates that the feedforward quantities $i_{o d}, i_{o q}$ and cross decoupled quantities $w \cdot C_{f} \cdot u_{o q}$ and $w \cdot C_{f} \cdot u_{o d}$ are used to achieve independent voltage control in the $d-q$ axis. To eliminate the voltage coupling, the outer voltage control loop is designed as Eqns. (11)-(12) [29].

$$
\begin{aligned}
& i_{d}=\left(K_{p u}+\frac{K_{I u}}{s}\right) \cdot\left(u_{o d}^{*}-u_{o d}\right)-w \cdot C_{f} \cdot u_{o q}+i_{o d} \\
& i_{q}=\left(K_{p u}+\frac{K_{I u}}{s}\right) \cdot\left(u_{o q}^{*}-u_{o q}\right)+w \cdot C_{f} \cdot u_{o d}+i_{o q}
\end{aligned}
$$

Applying Eqns. (11)-(12) into Eqns. (5)-(6), the following are obtained:

$$
\begin{aligned}
& s \cdot C_{f} \cdot u_{o d}=\left(K_{p u}+\frac{K_{I u}}{s}\right) \cdot\left(u_{o d}^{*}-u_{o d}\right) \\
& s \cdot C_{f} \cdot u_{o q}=\left(K_{p u}+\frac{K_{I u}}{s}\right) \cdot\left(u_{o q}^{*}-u_{o q}\right)
\end{aligned}
$$

$u_{o d}$ and $u_{o q}$ can be controlled separately without coupling.

\section{B. Adaptive Droop Control}

The values of the active and reactive powers flowing between two AC voltage sources, which are connected in parallel through the line impedance as shown in Fig. 2, can be calculated as Eqns. (15)-(16) [30], [31]. In Fig. 2, $R$ and $X$ are the line impedance and inductance, respectively. $U_{1}$ and $U_{2}$ are the Root-Mean-Square (RMS) value of the AC voltage sources, and their phases are $\theta_{1}$ and $\theta_{2}$, respectively. If $X \gg>$ and $\delta=\theta_{1}-\theta_{2}$ is small, then Eqns. (15)-(16) can be simplified as Eqns. (17)-(18).

$$
\begin{gathered}
P=\frac{U_{1}}{R^{2}+X^{2}}\left[R\left(U_{1}-U_{2} \cos \delta\right)+X U_{2} \sin \delta\right] \\
Q=\frac{U_{1}}{R^{2}+X^{2}}\left[-R U_{2} \sin \delta+X\left(U_{1}-U_{2} \cos \delta\right)\right] \\
P=\frac{U_{1} U_{2}}{X} \delta \\
Q=\frac{U_{1}^{2}-U_{1} U_{2}}{X}
\end{gathered}
$$

As shown by Eqns. (17)-(18), the active power, flowing from voltage source 1 to 2 through a highly inductive line impedance, can be controlled by varying the phase $\delta$. The reactive power supplied by source 1 can be controlled by controlling the magnitude of source $1\left(U_{1}\right)$. This is the basis of the conventional $P$ vs. $f$ and $Q$ vs. $U$ droop controls. As 


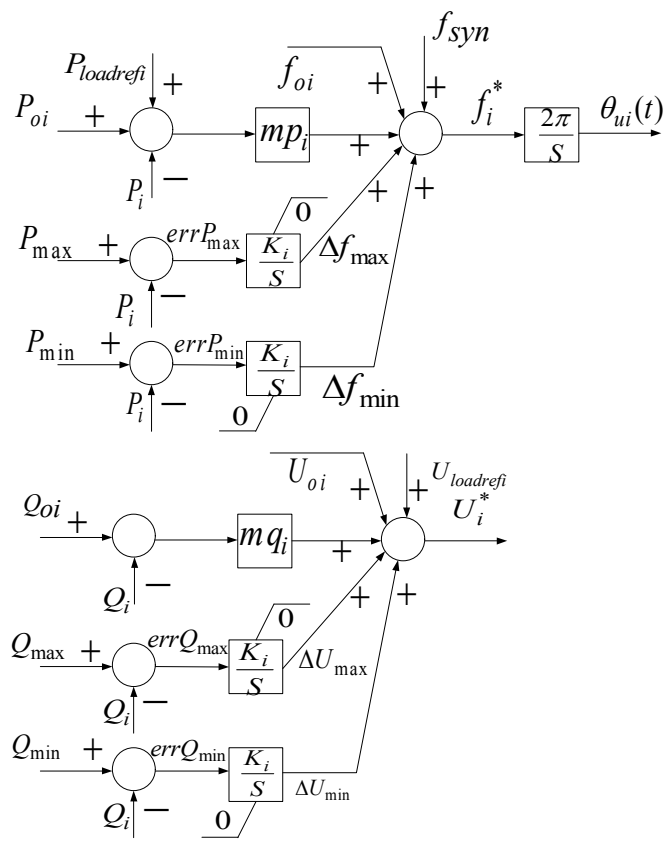

Fig. 3. Adaptive $P$ vs. $f$ and $Q$ vs. $U$ droop control diagram.

shown in Eqns. (19)-(20), in most cases, $f_{o i}$ and $U_{o i}$ are the nominal values [17].

$$
\begin{gathered}
f_{i}^{*}=f_{o i}+m p_{i}\left(P_{o i}-P_{i}\right) \\
U_{i}^{*}=U_{o i}+m q_{i}\left(Q_{o i}-Q_{i}\right)
\end{gathered}
$$

Where, $i$ is the DG index, $m p_{i}$ and $m q_{i}$ are the droop parameters, and $U_{i}, f_{i}, P_{i}$, and $Q_{i}$ are the bus RMS voltage, the system frequency, and the locally measured active and reactive powers, respectively. The subscript $o$ represents the preset values of the normal operating points.

The angle $\delta$ is generated by dynamically controlling the frequency of source 1 . The relationship between the frequencies of the two interconnected $\mathrm{AC}$ voltage sources and $\delta$ is given by Eq. (21)

$$
\delta=2 \pi \int\left(f_{1}-f_{2}\right) d t
$$

For directly coupled DG units, the impedance between two DGs is just the low voltage distribution feeder and has the relationship $R>>$ [32], [33]. Eqns. (15)-(16) can then be simplified as Eqns. (22)-(23). The droop can be based on $f(P)$ as in Eq. (19) or reversely, $P(f)$, where the frequency is measured and drooped to determine $P$. Unlike measurements of power, the accurate measurement of frequency is difficult. Therefore, the $f(P)$ strategy is often chosen [34]. In this paper, the $f(P)$ strategy is applied with frequency as a function of the delivered active power.

$$
\begin{gathered}
P=\frac{U_{1}^{2}-U_{1} U_{2}}{R} \\
Q=-\frac{U_{1} U_{2}}{R} \delta
\end{gathered}
$$

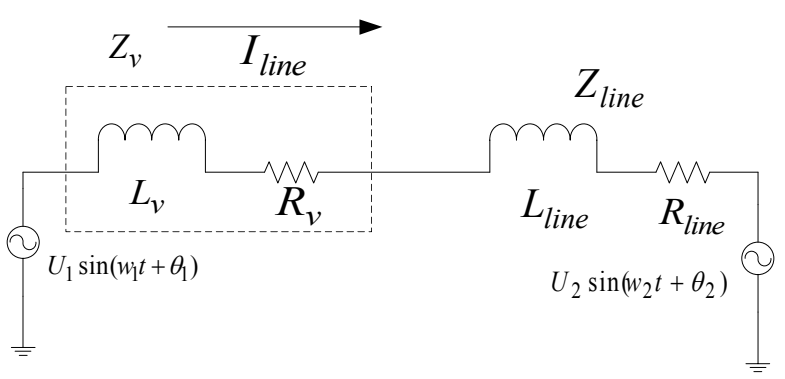

Fig. 4. Virtual impedance in series with the real line impedance.

In order to prevent the inverter from filling up beyond its capacity, the problem of reaching the maximum output power needs to be addressed. It is possible to take advantage of two assumptions: 1) the inverter can transiently sustain overshoots of the value $P_{\max }$ for short periods of time; 2) the inverter can transiently sustain the condition of $P<0$ for brief periods of time.

Fig. 3 show the proposed $P$ vs. $f$ and $Q$ vs. $U$ droop control diagrams. In Fig. 3, $P_{\max }$ is the allowed maximum output power, $P_{i}$ is the output active power of the $i$ th inverter, and $\operatorname{err} P_{\max }$ is the value of $P_{\max }$ minus $P_{\min }$. If the power is larger than its maximum, $\operatorname{err} P_{\max }$ is negative and the integral starts generating a negative value for the offset. This offset in turns translates the droop curve down. It keeps on translating down until the offset exactly matches the value where $\operatorname{err} P_{\max }=0$. Until it does, the integral keeps on increasing the offset. When power output matches the maximum power, the error become zero and the integral stops increasing the offset. As a result, a new steady state is reached with the offset $\Delta f_{\max }$. When preventing the injections of power from exceeding the minimum value, the approach used to calculate the offset $\Delta f_{\min }$ is very similar to the approach used to calculated $\Delta f_{\max }$. To prevent the reactive power from reaching the maximum or minimum values, the same strategy is used, as shown in Fig. 3.

As shown previously, for directly coupled DG units, the impedance between two DGs is just the low voltage distribution feeder and it has the relationship $R>X$. If the $f(\mathrm{P})$ strategy is to be used, a physical coupling inductor or a virtual coupling inductor should be added between the inverter terminals. The physical coupling inductor increases the size and weight of the system. The virtual impedance creates a "voltage drop" without generating real active and reactive powers losses and it is insensitive to the nature of the line impedance [31]. These physical inductances can be replaced by virtual impedances [1] and consecutive virtual impedance variations [30], [35], [36]. Therefore, a virtual impedance loop is added to the real line impedance as shown in Fig. 4. 


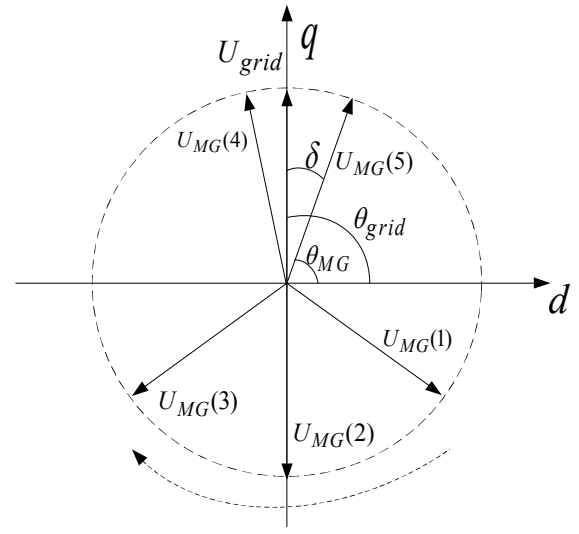

Fig. 5. Voltage vector synchronization process.

In Fig. $4, Z_{v}$ is the virtual impedance, $R_{v}$ is the resistive part of the virtual impedance, $L_{v}$ is the inductive part of the virtual impedance, $Z_{\text {line }}$ is the line impedance, $R_{\text {line }}$ is the resistive part of the line impedance, and $L_{\text {line }}$ is the inductive part of the line impedance.

\section{Synchronization Conditions and Frequency Restoration of Droop Controlled MGs}

The grid voltage $U_{\text {grid }}$ rotates at $50 \mathrm{~Hz}$ in the counter clockwise direction, while the MG Voltage $U_{M G}$ rotates at a frequency lower than $50 \mathrm{~Hz}$ in the same direction. If the vectors are strobed 50 times a second, the vector $U_{\text {grid }}$ stays still, while vector $U_{M G}$ draws back in the clockwise direction [2]. Fig. 5 shows the voltage vector synchronization process of $U_{\text {grid }}$ and $U_{M G}$. As Fig. 2 shows, if the grid injects the active power of $P_{\text {steady }}$ to the MG in the steady state, then from Eq. (17), the time to connect the MG to the grid is when $\delta$ satisfies Eq. (24).

$$
\delta=\frac{P_{\text {steady }} X}{U_{\text {grid }} U_{M G}}
$$

For droop control methods, the inherent trade-off between power sharing and voltage and frequency regulation is one of its drawbacks. In this paper, for the application of the voltage and frequency restoration and DG status identification, a MAS based global information discovery algorithm is used to acquire the global information. This algorithm is described in section III. There are two kinds of global information to be discovered: status information (such as the state of each DG and the weight factor to dispatch an active load) and performance metric (such as the average voltage and the output active power of a MG).

The frequency and voltage need to be restored only if the MG is operating in the island mode. In the grid-connected mode, the frequency and voltage are maintained by the main grid. In the island mode, it is assumed that, the DGs in the
MG are all running properly and within the upper and lower limits.

From Fig. 3, the $P$ vs. $f$ and $Q$ vs. $\mathrm{U}$ droop curves can be derived as Eqns. (25)-(26).

$$
\begin{gathered}
f_{i}^{*}=f_{o i}+m p_{i}\left(P_{o i}-P_{i}+P_{\text {loadrefi }}\right)+f_{s y n} \\
U_{i}^{*}=U_{o i}+m q_{i}\left(Q_{o i}-Q_{i}\right)+U_{\text {loadrefi }}
\end{gathered}
$$

$P_{o i}$ and $Q_{o i}$ are set to be zero, in this paper. In the island mode, $f_{s y n}$ is zero too, and the function of $f_{s y n}$ is described later. Then Eq. (25) can be rewritten as Eq. (27).

$$
\Delta f_{i}=f_{i}^{*}-f_{o i}=m p_{i}\left(P_{\text {loadrefi }}-P_{i}\right)
$$

Assume that:

$$
\begin{aligned}
& P_{\text {total }}=\sum_{i=1}^{n} P_{i} \\
& \lambda_{\text {total }}=\sum_{i=1}^{n} \lambda_{i}
\end{aligned}
$$

Where, $n$ is the number of DGs running in the MG, and $\lambda_{i}$ is the weight factor to share all of the active power consumed by the load, which is decided based on the capacity or the running cost of the DGs.

If:

$$
P_{i}=P_{\text {total }} \cdot \lambda_{i} / \lambda_{\text {total }}
$$

Then:

$$
P_{\text {loadrefi }}=P_{\text {total }} \cdot \lambda_{i} / \lambda_{\text {total }}+\Delta f_{i} / m p_{i}
$$

After restoration of the frequency, $\Delta f_{i}$ is zero. Thus, $P_{\text {loadrefi }}$ is rewritten as Eq. (32).

$$
P_{\text {loadrefi }}=P_{\text {total }} \cdot \lambda_{i} / \lambda_{\text {total }}
$$

Through the global information discovery process implemented by the MAS (as mentioned before), $P_{\text {total }}$ and $\lambda_{\text {total }}$ can be acquired by all of the DGs in the MG. $\lambda_{i}$ is a local parameter, which can be adjusted on line.

The frequency of the MG is restored by $P_{\text {loadrefi }}$ in the island mode. In order to change to the grid-connected mode from the island mode, the synchronization conditions need to be met. Therefore, $f_{s y n}$ is used to find a suitable angle $\delta$ to connect the MG to the main grid. $f_{s y n}$ is set to be a small value ( $0.2 \mathrm{~Hz}$ for example) when the $\mathrm{MG}$ going to connect to the main grid. At other times, it is set to be zero. When $f_{s y n}$ is bigger, it is able to find a suitable time to connect to the main grid more quickly, as described in Fig. 5. However, it must be lower than $0.5 \mathrm{~Hz}$ to satisfy the standard of frequency variation.

Unlike the frequency, the voltage of a MG is not a global parameter [37]. The frequencies of all of the DGs are equal in 


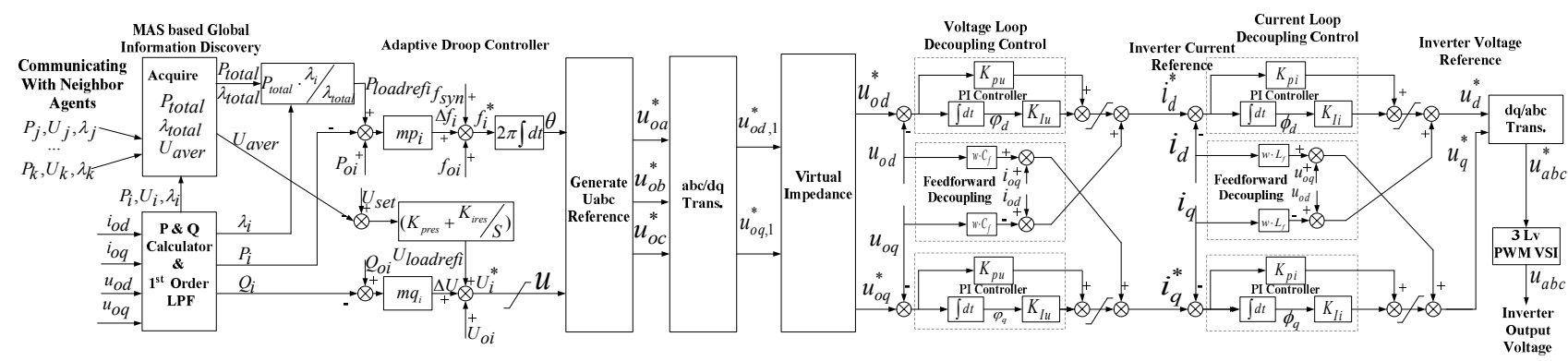

Fig. 6. Control block for inverter-interfaced DGs.

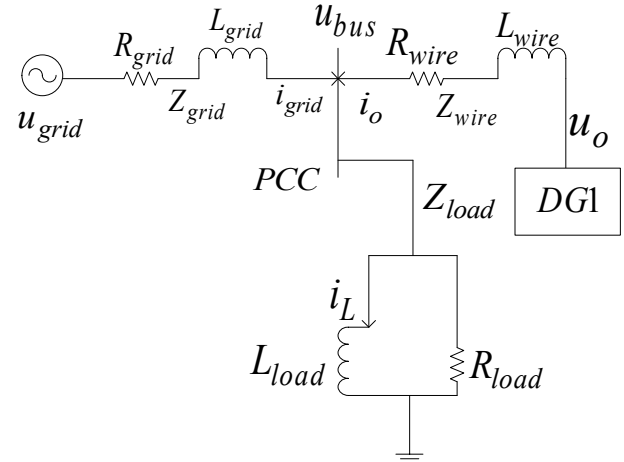

Fig. 7. Microgrid equivalent circuit model.

the steady state, but the amplitudes and phase angles of the voltages for the DGs are different from each other. In order to restore the voltage, a global information discovery algorithm is implemented based on a MAS to derive the average voltage $U_{\text {aver }}$ of the DGs. A proportional-integral (PI) controller is used to calculate $U_{\text {loadrefi }}$ and to restore the average voltage of the MG to its preset value. The relationship between $U_{\text {aver }}$ and $U_{\text {loadrefi }}$ is given by Eqns. (33)-(34).

$$
\begin{gathered}
U_{\text {aver }}=\sum_{i=1}^{n} U_{i} / n \\
U_{\text {loadrefi }}=\left(U_{\text {set }}-U_{\text {aver }}\right) \cdot\left(K_{\text {pres }}+K_{\text {ires }} / S\right)
\end{gathered}
$$

Where, $K_{\text {pres }}$ and $K_{\text {ires }}$ are the PI controller parameters used to restore the average voltage of the MG.

\section{Small-Signal Model Based Stability Analysis of a Microgrid System}

Fig. 6 shows a control block diagram of inverter interfaced DGs in the $d-q$ rotating reference frame. As shown in Fig. 6 , the DG controllers involve three types of controllers: the droop controllers, the voltage controllers and the current controllers. The droop controller calculates the output voltage reference, whereas the voltage and current controllers control the inverter voltage and current, respectively. As described in section II, voltage controllers and current controllers are all PI controllers.

From Fig. 6, the mathematical equations are derived as Eqns. (35)-(42).

$$
\begin{gathered}
\dot{\phi}_{d}=i_{d}^{*}-i_{d} \\
\dot{\phi}_{q}=i_{q}^{*}-i_{q} \\
u_{d}^{*}=u_{o d}+K_{p i} \cdot\left(i_{d}^{*}-i_{d}\right)+K_{I i} \cdot \phi_{d}-w \cdot L_{f} \cdot i_{q} \\
u_{q}^{*}=u_{o q}+K_{p i} \cdot\left(i_{q}^{*}-i_{q}\right)+K_{I i} \cdot \phi_{q}+w \cdot L_{f} \cdot i_{d} \\
\dot{\varphi}_{d}=u_{o d}^{*}-u_{o d} \\
\dot{\varphi}_{q}=u_{o q}^{*}-u_{o q} \\
i_{d}^{*}=i_{o d}+K_{p u} \cdot\left(u_{o d}^{*}-u_{o d}\right)+K_{I u} \cdot \varphi_{d}-w \cdot C_{f} \cdot u_{o q} \\
i_{q}^{*}=i_{o q}+K_{p u} \cdot\left(u_{o q}^{*}-u_{o q}\right)+K_{I u} \cdot \varphi_{q}+w \cdot C_{f} \cdot u_{o d}
\end{gathered}
$$

Fig. 7 shows a MG equivalent circuit model. The mathematical equations of the $\mathrm{MG}$ equivalent circuit can be obtained from Fig. 7, as Eqns. (43)-(50).

$$
\begin{gathered}
L_{\text {wire }} \cdot \dot{i}_{\text {od }}=u_{\text {od }}-u_{\text {bus }, d}-R_{\text {wire }} \cdot i_{o d}+w \cdot L_{\text {wire }} \cdot i_{o q} \\
L_{\text {wire }} \cdot \dot{i}_{o q}=u_{o q}-u_{\text {bus }, q}-R_{\text {wire }} \cdot i_{o q}-w \cdot L_{\text {wire }} \cdot i_{o d} \\
L_{\text {grid }} \cdot \dot{i}_{\text {grid }, d}=u_{\text {grid }, d}-u_{\text {bus }, d}-R_{\text {grid }} \cdot i_{\text {grid }, d}+w \cdot L_{\text {grid }} \cdot i_{\text {grid }, q} \\
L_{\text {grid }} \cdot \dot{i}_{\text {grid }, q}=u_{\text {grid }, q}-u_{\text {bus }, q}-R_{\text {grid }} \cdot i_{\text {grid }, q}-w \cdot L_{\text {grid }} \cdot i_{\text {grid }, d} \\
L_{\text {load }} \cdot \dot{i}_{l, d}=u_{\text {bus }, d}+w \cdot L_{\text {load }} \cdot i_{l, q} \\
L_{\text {load }} \cdot \dot{i}_{l, q}=u_{\text {bus }, q}-w \cdot L_{\text {load }} \cdot i_{l, d} \\
u_{\text {bus }, d}=R_{\text {load }} \cdot\left(i_{\text {od }}+i_{\text {grid }, d}-i_{l, d}\right) \\
u_{\text {bus }, q}=R_{\text {load }} \cdot\left(i_{o q}+i_{\text {grid }, q}-i_{l, q}\right)
\end{gathered}
$$

Because the PWM circuits and output filters have much faster dynamics than the inverter output controller, it is reasonable to assume that the actual inverter output voltage can follow the inverter voltage reference fast enough [17]. Thus, Eqns. (51)-(52) can be derived.

$$
\begin{aligned}
& u_{d}^{*}=u_{d} \\
& u_{q}^{*}=u_{q}
\end{aligned}
$$

From Eqns. (3)-(6) and Eqns. (35)-(52), a small-signal closed-loop model can be obtained as shown in Eqns. (53)-(54).

$$
\begin{aligned}
& \dot{x}=A \cdot \tilde{x}+B \cdot u \\
& y=C \cdot \tilde{x}+D \cdot u
\end{aligned}
$$

Where:

$$
\begin{aligned}
\tilde{x}= & {\left[\Delta i_{o d}, \Delta i_{o q}, \Delta i_{d}, \Delta i_{q}, \Delta u_{o d}, \Delta u_{o q}, \Delta i_{\text {grid }, d}, \Delta i_{\text {grid }, q}, \Delta i_{l, d}, \Delta i_{l, q},\right.} \\
& \left.\Delta \phi_{d}, \Delta \phi_{q}, \Delta \varphi_{d}, \Delta \varphi_{q}\right]^{T}
\end{aligned}
$$




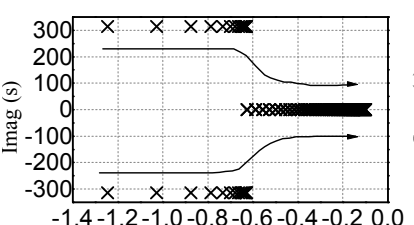

(a) Real (s)
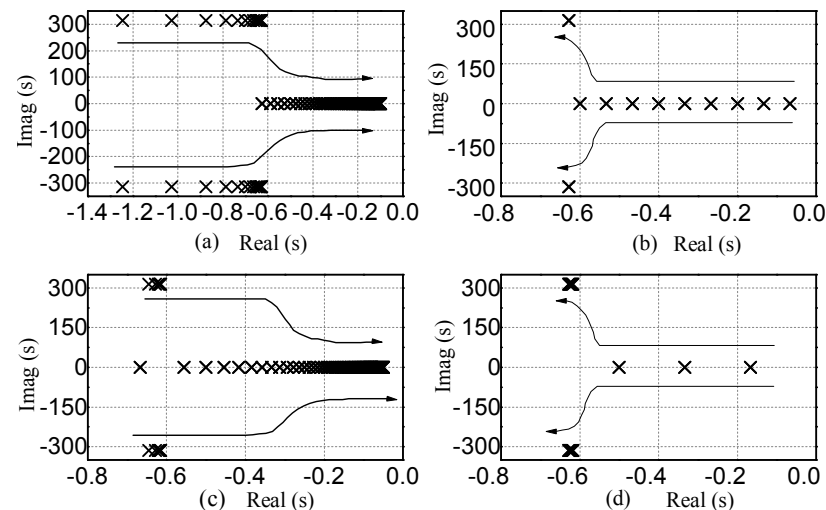

Fig. 8. Closed-loop eigenvalue analysis. (a) Dominant pole locus when $K_{p i}$ varies $\left(K_{I i}=10, K_{p u}=3, K_{I u}=10\right)$; (b) Dominant pole locus when $K_{I i}$ varies $\left(K_{p i}=15, K_{p u}=3, K_{I u}=10\right)$; (c) Dominant pole locus when $K_{p u}$ varies $\left(K_{p i}=15, K_{I u}=10\right.$, $\left.K_{I i}=10\right)$; (d) Dominant pole locus when $K_{I u}$ varies $\left(K_{p i}=15\right.$, $\left.K_{p u}=3, K_{I i}=10\right)$.

$$
\begin{aligned}
& u=\left[\Delta w, \Delta_{o d}^{*}, \Delta_{o q}^{*}, \Delta u_{\text {grid }, d}, \Delta u_{\text {grid }, q}\right]^{T} ; \\
& y=\left[\Delta u_{o d}^{*}-\Delta u_{o d}, \Delta u_{o q}^{*}-\Delta u_{o q}, \Delta i_{d}^{*}-\Delta i_{d}, \Delta i_{q}^{*}-\Delta i_{q}\right]^{T} .
\end{aligned}
$$

Fig. 8 shows the close-loop dominant eigenvalue locus when the PI parameters change. As shown in Fig. 8 (a), the dominant complex poles move to the right when increasing $K_{p i}$ from 1 to 100 while the other parameters are fixed. When $K_{p i}$ is smaller than 15 , the system is under damped. On the other hand, when $K_{p i}$ is bigger than 15, the poles are near the imaginary axis. As a result, the stability margin is lower. Fig. 8 (b) shows that the dominant complex poles move to the left when increasing $K_{I i}$ from 1 to 100 while the other parameters are fixed. This makes the system quicker but more oscillatory. Fig. 8 (c) indicates that the dominant complex poles move to the right when increasing $K_{p u}$ from 1 to 100 while the other parameters are fixed. When $K_{p u}$ is smaller than 3 , the system is under damped. On the other hand, when $K_{p u}$ is bigger than 3, the poles are near the imaginary axis. Thus, the stability margin is lower. Fig.8 (d) shows that the dominant complex poles move to the left when increasing $K_{I u}$ from 1 to 100 while the other parameters are fixed. This makes the system quicker but more oscillatory. This helps us to find suitable parameters for the inverter controllers.

\section{MAS-BASED GLOBAL INFORMATION DISCOVERY ALGORITHM}

As described in section II, the traditional droop control has the drawback of a trade-off between power sharing and voltage and frequency regulation. Several proposed

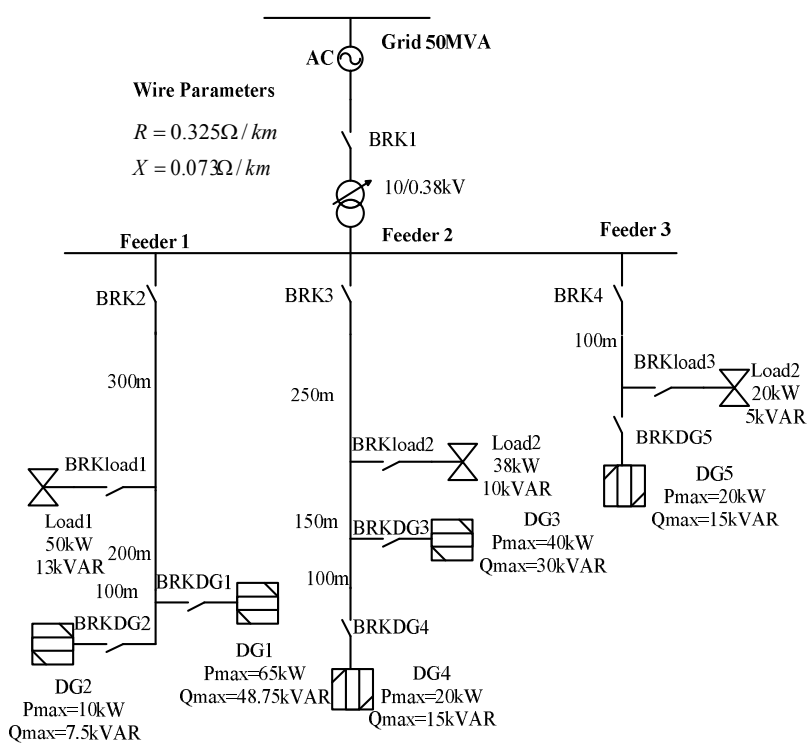

Fig. 9. Topology of a microgrid.

restoration controls must be carried out by means of a central controller, which requires some form of communication between the restoration control and the DGs, which is complex and not reliable for a single point of failure.

In section II, an adaptive droop control method is proposed. This method can restore the voltage and frequency. It can also properly dispatch the active power by using the status information (such as the state of each DG and the weight factor to dispatch the active load) and the performance metric (such as the average voltage and the output active power of the MG). This information is discovered by a MAS based global information discovery algorithm.

In recent years, global information discovery algorithms based on the MAS have attracted a great deal of interest in various areas [26], [38], [39]. These algorithms are based on multi agent coordination and do not need a powerful central controller. Thus, they are robust to the single point failures.

A MG, as shown in Fig. 9, is taken for research. The MG is coupled with the main grid through the PCC, and can operate either in the island mode or the grid-connected mode. There are three feeders in the MG. Feeder 1 has one load and two DGs; Feeder 2 also has one load and two DGs; while feeder 3 has one load and one DG. The length and parameters of the transmission line, the capacities of the DGs and the load demand are shown in Fig. 9. In this paper, it is assumed that each DG unit in the MG is assigned with an agent. Every agent knows the local information (the agent can measure the information locally and acquire it without any communication), such as the local voltage, the output active power of a certain DG, and so on. However, it does not have direct access to the global information (this information cannot be acquired directly, by communicating with the corresponding neighbor agents, each agent must acquire the global information through the MAS-based global 
TABLE I

Data of Each Agent to Communicate

\begin{tabular}{lllllll}
\hline No. & $\begin{array}{l}\text { Neighb_ } \\
\text { ors }\end{array}$ & Syn & $\begin{array}{l}P_{i} \\
(\mathrm{~kW})\end{array}$ & $\begin{array}{l}V_{i} \\
(\mathrm{~V})\end{array}$ & $\begin{array}{l}\lambda_{i} \\
\text { DGs' statee } \\
\text { vector }\end{array}$ \\
\hline 1 & 2,3 & 1 & 47.8 & 381.5 & 0.45 & {$[1,0,0,0,0]$} \\
2 & 1,4 & 0 & 6.4 & 381.8 & 0.06 & {$[0,1,0,0,0]$} \\
3 & $1,4,5$ & 0 & 26.5 & 379.0 & 0.25 & {$[0,0,3,0,0]$} \\
4 & $2,3,5$ & 0 & 15.9 & 380.4 & 0.15 & {$[0,0,0,4,0]$} \\
5 & 3,4 & 0 & 9.5 & 372.4 & 0.09 & {$[0,0,0,0,5]$} \\
\hline
\end{tabular}

information discovery algorithm), such as the average voltage of the MG, the total output active powers of all of the DGs, and so on. As Fig. 6 shows, with the local information of $P_{i}$, $U_{i}$, and $\lambda_{i}$, agent $i$ can acquire the global information of $P_{\text {total }}, U_{\text {aver }}, \lambda_{\text {total }}$ by communicating with its neighbors $j$ and $k$. With this global information, $P_{\text {loadref }}$ and $U_{\text {aver }}$ can be calculated by Eq. (32) and Eq. (34), respectively. The adaptive droop controller can restore

the frequency and voltage deviation of the MG with $P_{\text {loadref }}$ and $U_{\text {aver }}$. This overcomes the main drawback of traditional droop control methods (the trade-off between power sharing and voltage and frequency regulation).

In order to improve the robustness against communication failures, the topology of the communication network is designed to satisfy the $N-1$ rule, which means that the rest of the communication network can work normally, with the breakdown of any one agent. Each agent can communicate with its neighbor agents. For example, in Fig. 9, DG 1 can communicate with DG 2 and DG 3. All of the agents constitute a multi agent system. The original data of each DG for the case study are summarized in Table I.

In the global information discovery algorithm, the information discovery process for the $i$ th agent is represented as Eq. (55).

$$
x_{i}^{k+1}=x_{i}^{k}+\sum_{j \in N_{i}} a_{i j}\left(x_{j}^{k}-x_{i}^{k}\right)
$$

Where, $x_{i}^{k}$ and $x_{j}^{k}$ are the pieces of information (average voltage, average active power, and so on) discovered by agents $i$ and $j$ at iteration $k$, respectively; $i=1,2, \ldots, n$; $n$ is the number of agents in the MG; $x_{i}^{k}$ is updated to $x_{i}^{k+1}$ at iteration $k+1 ; a_{i j}$ is the coefficient for the information exchanged between the neighboring agents $i$ and $j$; and $N_{i}$ is the set of neighbors for agent $i$.

The coefficient $a_{i j}$ plays an important role for the convergence speed. Y. Xu and W. Liu summarize and discuss several methods for designing the coupling coefficients [25]. $a_{i j}$ is updated based on Eq. (56), as proposed in [25].

$$
a_{i j}=\left\{\begin{array}{cl}
\frac{2}{\left(n_{i}+n_{j}+\varepsilon\right)} & j \in N_{i} \\
1-\sum_{j \in N_{i}} \frac{2}{\left(n_{i}+n_{j}+\varepsilon\right)} & i=j \\
0 & \text { otherwise }
\end{array}\right.
$$

Where, $n_{i}$ is the number of neighbors for agent $i, n_{j}$ is the number of neighbors for agent $j$, and $N_{i}$ is the set of neighbors for agent $i . \varepsilon$ is a very small number. The value of $\varepsilon$ is set to 0.3 in this paper. Based on the consensus theory, the overall information discovery process can be modeled as a discrete time linear system as shows in Eq. (57).

$$
X^{k+1}=D \cdot X^{k}
$$

Where, $X^{k}=\left[x_{1}^{k}, x_{2}^{k}, \ldots, x_{n}^{k}\right]^{T}$ is the vector of the discovered information at the $k$ th iteration; and $D$ is the Laplacian matrix of the multiagent network graph [39]. The convergence speed is determined by the second largest eigenvalue of $D$.

The global information discovery algorithm can guarantee the convergence for systems of any size and topology, as shown in [25], [27]. In this paper, the information discovery process terminates once the following Equation is valid:

$$
\left|x_{i}^{k+1}-x_{i}^{k}\right|<\gamma
$$

Where, $\gamma$ is a small real number that defines the precision requirement. The value of $\gamma$ is set to 0.01 in this paper.

For the application of the voltage and frequency restoration and the DG status identification, there are two kinds of information to be discovered: the status information and the performance metric [40]. In order to acquire information on the number of DGs and which DG are running in this $\mathrm{MG}$, each agent in the $\mathrm{MG}$ is assigned a unique state vector beforehand. In the beginning, agent $i$ 's $N$-dimensional ( $N$ is the total number of agents in the MG and it is a constant for a given $\mathrm{MG}$ ) state vector $s_{i}$ has only one nonzero element at $(i, 1)$ with the value of $i$, that is $s_{i}=[0,0, \ldots, \mathrm{i}, \ldots, 0]^{T} \quad[27]$. The $i$ th elements of the state vector will converge to $i / M$ ( $M$ is the total number of working agents in the $\mathrm{MG}, M \leq N)$, by applying the global information discovery algorithm to the same elements of the state vectors, as shown in Fig. 10(e). When DG 4 is disconnected (but agent 4 is still working normally), agent 4's state vector $s_{4}$ is reset to $[0,0,0,0,0]^{T}$. By applying the global information discovery algorithm to the same elements of the state vectors, all of the elements except for the 4th element of the state vectors will converge to the same value $(i / M)$ as before, as can be seen in Fig. 10(f). By checking 


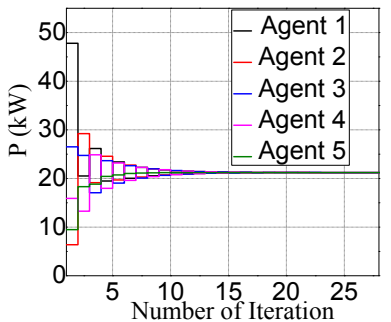

(a)

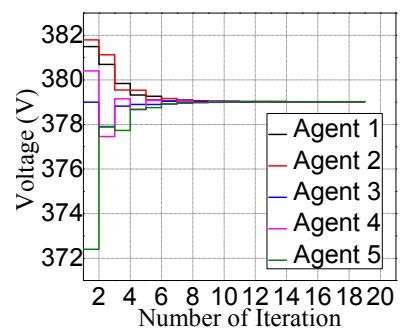

(c)

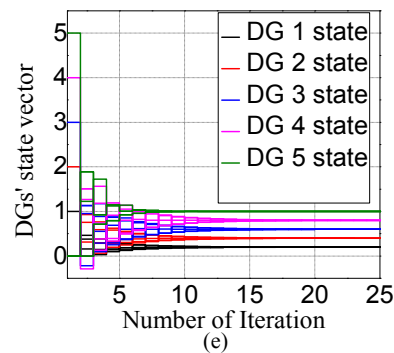

(e)

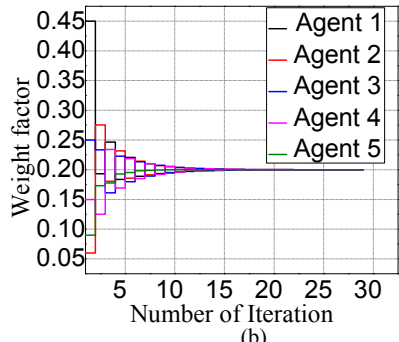

(b)
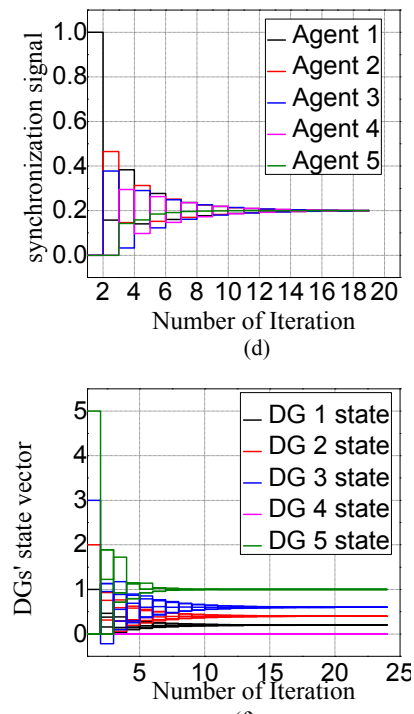

(f)
Fig. 10. Information discovery processes of status information and performance metrics. (a) Average active power discovery process. (b) Average weight factor discovery process. (c) Average voltage discovery process. (d) Synchronization signal discovery process. (e) DGs' state vector discovery process, when all DGs are connected to the MG. (f) DGs' state vector discovery process, when DG4 is disconnected from the MG.

the positions of the zeros, all of the agents will know that DG 4 is now disconnected. As a result, the number of DGs connected to the MG $n$ is updated. Furthermore, by comparing the $i$ th element with $x_{i} / M$, where $x_{i}$ can be either $i$ or 0 (when the corresponding DG is disconnected) and $M$ can be any integer between 1 and $N$, it can find the $M$ match of the converged state vector $\left[x_{1} / M, \ldots, x_{i} / M, \ldots, x_{N} / M\right]^{T}$. Thus, the number of working agents $M$ can also be found. Multiply the discovered average performance metrics by $M$, and the total value of the according metrics are derived.

In order to deal with the fault of an agent loss, all of the agents need send heartbeat packets to $i$ th's neighbors. Then each agent knows who its neighbors are and the number of neighbors at the present time. By using Eq. (56), $a_{i j}$ can be updated. When the MG is expanded, it only needs to update its' neighbors' information. Thus, the MAS control offers a plug and play performance which involves low costs related to modifications in the MG's architecture.
TABLE II

LOADS DATA SUMMARY

\begin{tabular}{llll}
\hline & Load 1 & Load 2 & Load 3 \\
\hline$R_{\text {load }}(\Omega)$ & 2.888 & 3.800 & 7.220 \\
$X_{\text {load }}(\Omega)$ & 11.108 & 14.440 & 28.880 \\
\hline
\end{tabular}

Agent 1 is responsible for synchronize with the main grid. Agent 1's synchronization signal Syn will be 1, when the micriogrid is going to change from the island mode to the grid-connected mode, otherwise Syn will be 0 . The other agents' synchronization signals $S y n$ are always initialized to be zero. Thus, when an agent finds that Syn converges to a nonzero value, $f_{s y n}$ in (25) is set to $0.2 \mathrm{~Hz}$ for all the droop controllers to synchronize the MG with the main grid, as described previously. The information discovery processes of the status information and the performance metrics are shown in Fig. 10.

Fig. 10(a) shows the average active power discovery process. All of the agents converge to the same value of $21.22=(47.8+6.4+26.5+15.9+9.5) / 5$ within 30 iterations. Fig. 10 (b) describes the average weight factor discovery process. It shows that all of the agents converge to the same value within 30 iterations. Fig. 10 (c) and Fig. 10 (d) indicate the average voltage and synchronization signal discovery processes, respectively. They also show that all of the agents converge to the same value within 30 iterations. Fig. 10 (e) shows the updating process of the $i$ th element of the $i$ th agents' state vectors when all of the DGs are connected to the MG. From Fig. 10 (e), it can be seen that all of the agents converge to the same value of $i / M$. Fig. 10 (f) shows the updating process of the $i$ th element of the $i$ th agents' state vectors when DG4 is disconnected from the MG. From Fig. 10 (f), it can be seen that all of the agents except for agent 4 converge to the same value of $i / M$ as before.

The data to communicate is represented by 16 bits for one piece of data. There are 9 pieces of data to communicate for each agent, and it is supposes that the maximum iteration to convergence is 50 for the global information discovery algorithm (which is rational considering the results in Fig. 10). Suppose that one agent has 10 neighbors as a maximum in the MG. In order to get the global information 5 times per second, the communicating rate should be higher than $16 \times 9 \times 50 \times 10 \times 5 \times 2=720 \mathrm{kbps}$. Nowadays, a communication rate of $720 \mathrm{kbps}$ is easy to fulfill. Thus, it is reasonable to get global information 5 times per second.

\section{SimUlation AND RESUlts}

The MG system model in Fig. 9 is simulated by using PSCAD/EMTDC. This model contains five inverter-interfaced DGs with PWM voltage source inverters. The DGs in the MG 
TABLE III

DGs DATA SUMMARY

\begin{tabular}{clllll}
\hline & DG 1 & DG 2 & DG 3 & DG 4 & DG 5 \\
\hline$P_{\max }(\mathrm{kW})$ & 65 & 10 & 40 & 20 & 20 \\
$Q_{\max }(\mathrm{kVar})$ & 48.75 & 7.5 & 30 & 15 & 15 \\
$V_{\max }(\mathrm{V})$ & 380 & 380 & 380 & 380 & 380 \\
$V_{\operatorname{mix}}(\mathrm{V})$ & 367 & 367 & 371.5 & 371.5 & 378.5 \\
$m p(\mathrm{~Hz} / \mathrm{kW})$ & 0.0077 & 0.0500 & 0.0125 & 0.025 & 0.025 \\
$m q(\mathrm{~V} / \mathrm{kVar})$ & 0.0770 & 0.5004 & 0.0818 & 0.1636 & 0.0289 \\
$\operatorname{Vir} \mathrm{X}(\Omega)$ & 0.2707 & 1.7598 & 0.4399 & 0.8799 & 0.8799 \\
\hline
\end{tabular}

are coordinated via $P$ vs. $f$ and $Q$ vs. $U$ adaptive droop controllers and the global information is discovered by a MAS.

All of the loads are connected in a star configuration and they can be connected and disconnected with a switch. The parameters of the loads in Fig. 9 are listed in Table II.

In this paper, the inverter is fed by a DC bus voltage source of $750 \mathrm{~V}$, which emulates the combined effects of a prime mover and energy storage. The carrier frequency of the VSI is $5000 \mathrm{~Hz}$. The rated power factors of the DGs are all set as 0.8 , and the minimum operating frequency is $49.5 \mathrm{~Hz}$, which is 0.5 $\mathrm{Hz}$ below the nominal frequency.

The fixed slops $m p_{i}$ of the $P$ vs. $f$ droop are chosen to be the value of the slop obtained for $P_{o i}=0$ in Eq. (25). They are list in Table III

When the value of the maximum offset expected for the voltage, $\Delta E$, is known, with the help of $Q_{\max }$, the slopes $m q_{i}$ of the $Q$ vs. $U$ droop can be determined by $\Delta E / Q_{\max }$. The maximum offset for the voltage is taken as the difference in voltage between the best and worst case scenarios [4]. The best case scenario is when the MG is fully energized from the grid, without micro sources or loads. The worst case scenario is when the MG is energized from the grid, without micro sources and with all of the loads. The voltages in the best case scenario are represented as $V_{\max }$, in the worst case scenario they are represented as $V_{\min }, V_{\max }, V_{\min }$, and the slopes $m q_{i}$ are list in Table III.

The virtual impedance can generate good system transient and steady-state responses if designed properly. The effects of virtual impedance have been verified in some published papers. The virtual impedance increases the system damping and enhances the system's transient response but it also affects the inverters' voltage regulation (which will cause voltage drop) [41], [42]. As Eq. (17) shows, the phase angle difference $\delta$ is affected by the coupling inductor $X$. The angle difference $\delta$ cannot be allowed to get too small to avoid errors caused by an imprecise measurement of $\delta$. However, in order to have a good linear approximation between $P$ and $\delta, \delta$ cannot be allowed to get too big. In
TABLE IV

OTHER PARAMETERS IN THIS PAPER

\begin{tabular}{llllllll}
\hline$L_{f}$ & $C_{f}$ & $K_{p i}$ & $K_{I i}$ & $K_{p u}$ & $K_{I u}$ & $K_{\text {pres }}$ & $K_{\text {ires }}$ \\
\hline $3 \mathrm{mH}$ & $34 \mathrm{uF}$ & 15 & 10 & 3 & 10 & 0.04 & 0.5 \\
\hline
\end{tabular}

this paper, the method to calculate the virtual impedance is from reference [2]. $\delta$ is set to be 7 degrees at full ratings. This guarantees operation in the linear region of the sinusoidal characteristic. This means that the virtual impedance $X$ can be calculated as Eq. (59) as shown in [2]. The Virtual impedances $X$ are calculated and listed in Table III.

$$
X=\frac{U_{1} U_{2}}{P_{\max }} \delta_{\max }
$$

The values of $K_{\text {pres }}$ and $K_{\text {ires }}$ in Eq. (34) are designed by experiential adjustments according to the simulation results. Within certain limits, when the value of $K_{\text {pres }}$ is bigger, the speed is higher for restoring the voltage. However, it cannot be too big to maintain system stability. Within certain limits, when the value of $K_{\text {ires }}$ is bigger, the steady state deviation is lower. However, it cannot be too big either to maintain system stability. The appropriate values of $K_{\text {pres }}$,

$K_{\text {ires }}$ and the other parameters used in this paper are given in Table IV.

In order to show the decoupling effect of the decoupling method introduced in section II-A, a comparison study was carried out. In the comparison study, the performance of the decoupling method is compared with the method without a feed-forward decoupling network. The results are shown in Fig. 11. As shown in the figure, when the reference voltages are given a step change at $0.1 \mathrm{~s}$, the decoupling method has a faster response speed and a lower steady state deviation than the method without a feed-forward decoupling network.

In order to verify the proposed adaptive droop control method, the simulation sequence was set as follows: 1) $0-2 \mathrm{~s}$, the MG is operating in the island mode and all of the DGs and loads are connected; 2) at $3 \mathrm{~s}$, DG 4 is disconnected; 3) $2-6.8 \mathrm{~s}, f_{s y n}$ is set to $0.2 \mathrm{~Hz}$ for all of the DGs to synchronize the MG with the main grid, in order to be connected to the main grid; 4) $6.8-13 \mathrm{~s}$, the MG is connected to the main grid, all of DGs are set to output $10 \mathrm{~kW}$ of active power and DG 1, DG 2, DG 3 and DG 5 are set to output $10 \mathrm{kVar}, 5 \mathrm{kVar}, 8 \mathrm{kVar}$ and $0 \mathrm{kVar}$ of reactive power, respectively; 5) $13-14 \mathrm{~s}$, it is assumed that the DGs have not detected the island to analyze the effect of the time delay to the system; 6) at $14 \mathrm{~s}$, all of the DGs get the information of the island, recover the frequency and dispatch active power; 7) at $17 \mathrm{~s}$, load 2 is disconnected. In this simulation, the global information is acquired once every 0.2 seconds. The simulation results of the MG are shown in Figs. 12-16.

Fig. 12 shows the active powers and frequencies of the DGs. 


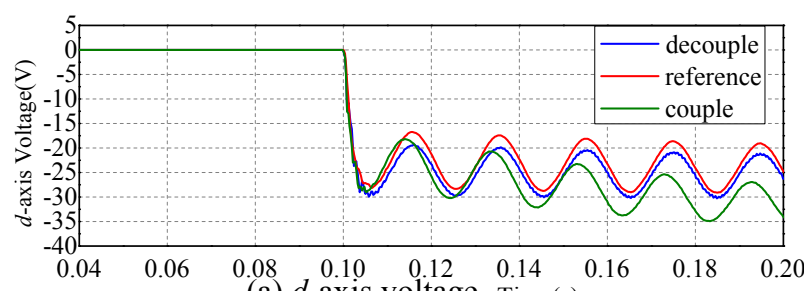

(a) $d$-axis voltage Time(s)

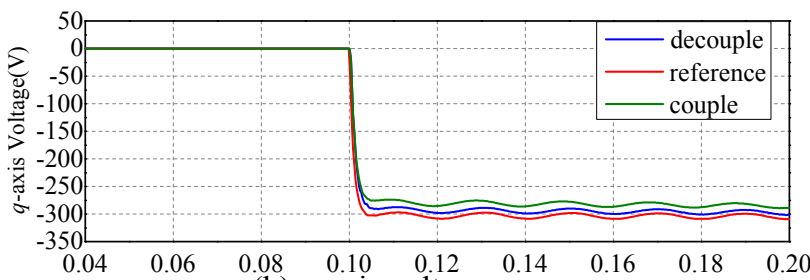

(b) $q$-axis voltage Time(s)

Fig. 11. Compare the feed-forward decoupling control methods with the coupling control methods.
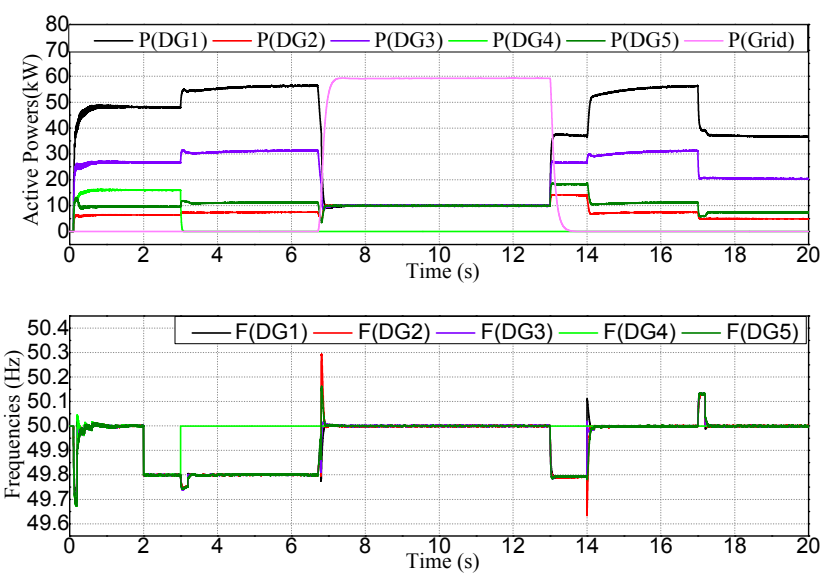

Fig. 12. Active powers and the frequencies of DGs.
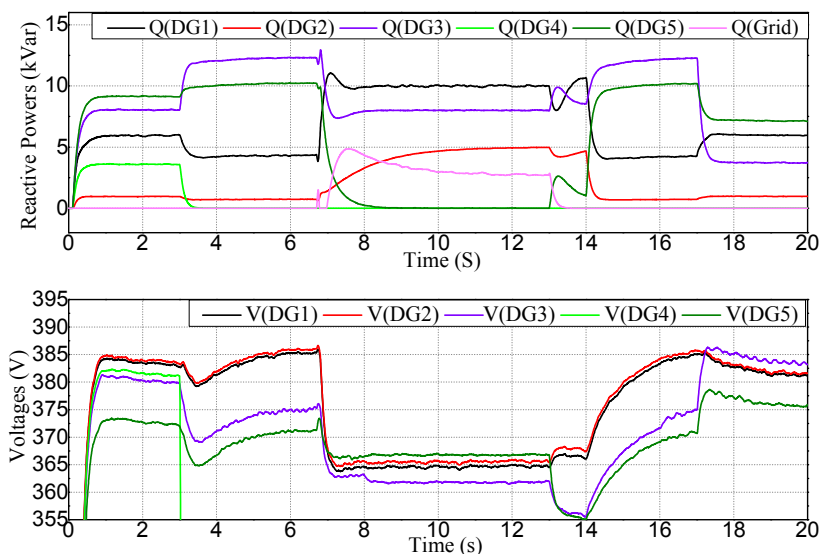

Fig. 13. Reactive Powers and Voltages of DGs.

In the island mode, the frequency can be restored and the active powers can be simultaneously dispatched as desired. In the grid-connected mode, the system frequency is provided by the main grid, the output active power of each of the DGs can follow up the preset value, while the grid provides the residual load demand. For the time delay of the island mode detection during $13-14 \mathrm{~s}$, the frequency is not restored but the system

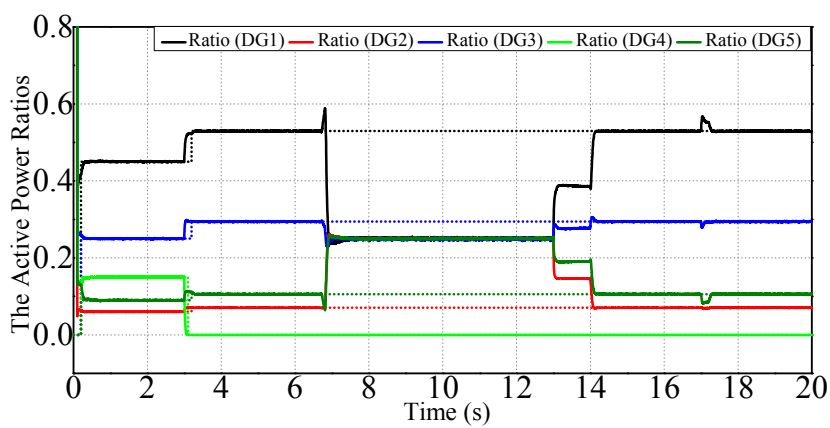

Fig. 14. Active power ratios of DGs.
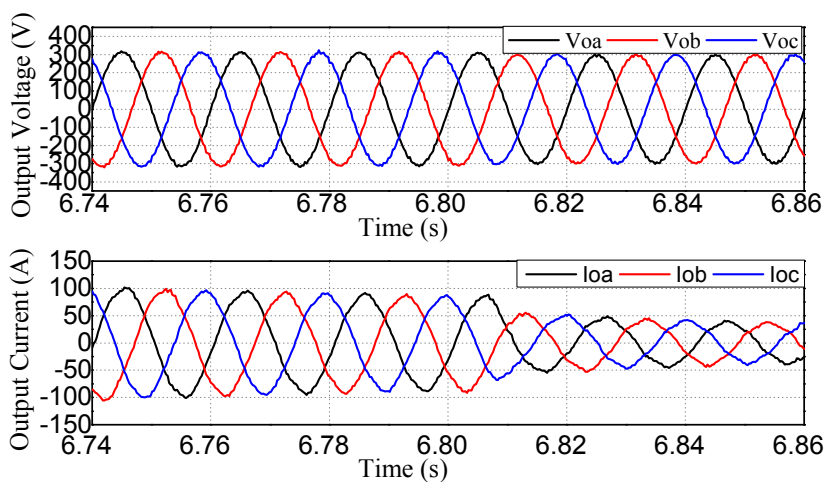

Fig. 15. Output voltage and current of DG1 when the microgrid shits to grid-connected mode from island mode.

can still work properly. At $17 \mathrm{~s}$, when load 2 is disconnected, all of the DGs will decrease the output active power while the frequency is higher than the normal value for a short time, then it is restored to the normal value.

Fig. 13 shows the reactive powers and voltages of the DGs. In the island mode, when DG 4 is disconnected at $3 \mathrm{~s}$, the voltages are decreased and restored for a while. In the grid-connected mode, the voltages are provided by the main grid and the output reactive power of each DG can follow up the preset value.

Fig. 14 shows the active power ratios for each of the DGs. The dashed line represents the preset ratios and the solid line represents the actual active power ratios. Only the moment when the MG is in the island mode needs to be seen, because in the grid-connected mode, each of the DGs is set to output $10 \mathrm{~kW}$ of active power. In this figure, it can be seen that the DGs' active power ratios can follow the preset values most of the time. During the time delay of the island mode detection of $13-14 \mathrm{~s}$ the actual active power ratios cannot follow the preset value. The difference between the actual ratios and actual ratios at other times is caused by the time delay of the global information discovery.

Fig. 15 and Fig. 16 show the output voltage and current of DG 1 when the microgrid connects to and disconnects from the main grid, respectively. As the reference values of the output active and reactive powers are changed between the island mode and the grid-connected mode in the study case, the magnitudes of the currents of $i_{o a}, i_{o b}$ and $i_{o c}$ changed 

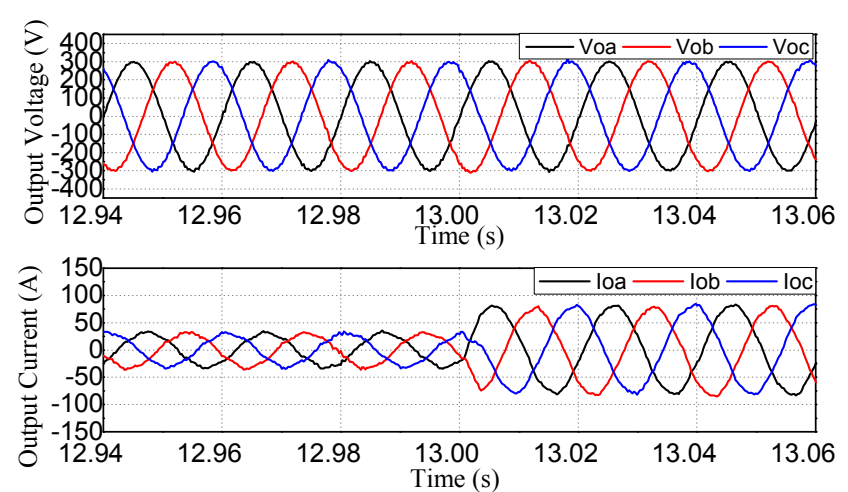

Fig. 16. Output voltage and current of DG1 when the microgrid shifts to island mode from grid-connected mode.
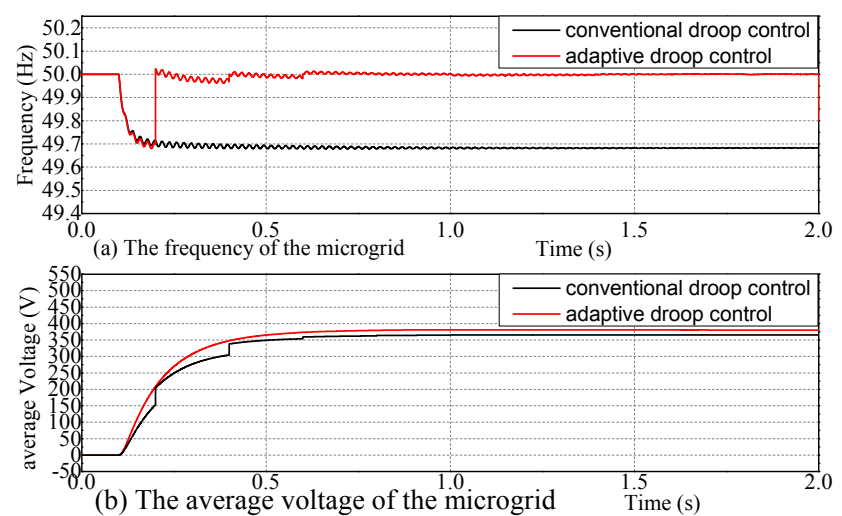

Fig. 17. Compare the adaptive droop control method with the conventional droop control method.

accordingly. In the steady state, the total harmonic distortions (THD) of DG1's currents are $0.81 \%$ and $2.30 \%$ when the microgrid is in the island mode and the grid-connected mode, respectively. The current THD value is smaller than the international standard [43] during the island and grid-connected operation modes.

In order to verify the validity of proposed adaptive droop control method, comparative results with conventional droop control methods are shown in Fig. 17. From Fig. 17, it can be seen that with the proposed adaptive droop control method, the microgrid can restore the frequency and voltage deviation. Meanwhile the microgrid controlled by the conventional droop control method had frequency and voltage deviations in the steady state.

\section{CONCLUSIONS}

In this paper, an adaptive droop control method is proposed for MGs. This adaptive droop controller can restore the voltage and frequency with global information. Meanwhile, the active power can be dispatched properly, according to the capacity or running cost of each DG. Furthermore, the MG can shift between the island mode and the grid-connected mode smoothly by using the proposed control method. The proposed adaptive droop control method can overcome the drawback of the trade-off between the power sharing and the voltage and frequency regulation experienced in the traditional droop control. By using a global information discovery algorithm based on the MAS, each agent only communicates with its direct neighbors, and the global information that is needed for each of the DGs in the MG can be acquired. The small-signal mode of the DGs in the MG is also set up. The proper current and voltage controller parameters for the inverter-interfaced DG can be found by using the small-signal mode. The simulation results demonstrate that the proposed method is effective.

\section{ACKNOWLEDGMENT}

This work was supported by the Natural Science Foundation of China under contact $(61100159,61233007)$, the National High Technology Research and Development Program of China (863 Program: 2011AA040103), Foundation of Chinese Academy of Sciences under contact (KGCX2-EW-104), financial support of the Strategic Priority Research Program of the Chinese Academy of Sciences under contact XDA06021100.

\section{REFERENCES}

[1] E. Planas, A. Gil-de-Murob, J. Andreua, I. Kortabarriaa, and I. M. de Alegríaa, "General aspects, hierarchical controls and droop methods in microgrids: A review," Renewable \& Sustainable Energy Reviews, Vol. 17, pp. 147-159, Jan. 2013.

[2] EETD http://eetd.lbl.gov/sites/all/files/publications/ctrl-de esign- microgrid-components.pdf, Jun. 20th 2014.

[3] R. Zamora and A. K. Srivastava, "Controls for microgrids with storage: Review, challenges, and research needs," Renewable and Sustainable Energy Reviews, Vol. 14, No. 7, pp. 2009-2018, Sep. 2010.

[4] K. Alanne and A. Saari, "Distributed energy generation and sustainable development," Renewable \& Sustainable Energy Reviews, Vol. 10, No. 6, pp. 539-558, Dec. 2006.

[5] MICROGRIDS http://microgrids.eu/micro2000/presentati ons/16.pdf, Jun. 13th 2014.

[6] J. Y. Huang, J. Chuanwen, and X. Rong, "A review on distributed energy resources and MicroGrid," Renewable \& Sustainable Energy Reviews, Vol. 12, No. 9, pp. 2472-2483, Dec. 2008.

[7] J. J. Justo, F. Mwasilu, J. Lee, and J.-W. Jung, "AC-microgrids versus DC-microgrids with distributed energy resources: a review," Renewable \& Sustainable Energy Reviews, Vol. 24, pp. 387-405, Aug. 2013.

[8] J. A. Peas Lopes, C. L. Moreira, and A. G. Madureira, "Defining control strategies for MicroGrids islanded operation," IEEE Trans. Power Syst., Vol. 21, No.2, pp. 916-924, May 2006.

[9] F. Katiraei, R. Iravani, N. Hatziargyriou, and A. Dimeas, "Microgrids management," IEEE Power Energy Mag., Vol. 6, No. 3, pp. 54-65, May 2008.

[10] T. S. Ustun, C. Ozansoy, and A. Zayegh, "Recent developments in microgrids and example cases around the world-a review," Renewable and Sustainable Energy Reviews, Vol. 15, No. 8, pp. 4030-4041, Oct. 2011.

[11] N. W. A. Lidula and A.D. Rajapakse, "Microgrids 
research: A review of experimental microgrids and test systems," Renewable \& Sustainable Energy Reviews, Vol. 15, No. 1, pp. 186-202, Jan. 2011.

[12] J. M. Guerrero, L.G. de Vicuna, J. Matas, J. Miret, and M. Castilla, "A wireless controller for parallel inverters in distributed online UPS systems," in Proc. IECON, pp. 1637-1642, 2003.

[13] E. Barklund, N. Pogaku, M. Prodanovic, C. Hernandez-Aramburo, and T. C. Green, "Energy management in autonomous microgrid using stability-constrained droop control of inverters," IEEE Trans. Power Electron., Vol. 23, No. 5, pp. 2346-2352, Sep. 2008.

[14] G. Diaz, C. Gonzalez-Moran, J. Gomez-Aleixandre, and A. Diez, "Scheduling of droop coefficients for frequency and voltage regulation in isolated microgrids," IEEE Trans. Power Syst., Vol. 25, No. 1, pp. 489-496, Feb. 2010.

[15] R. Majumder, B. Chaudhuri, A. Ghosh, R. Majumder, G. Ledwich, and F. Zare, "Improvement of stability and load sharing in an autonomous microgrid using supplementary droop control loop," IEEE Trans. Power Syst., Vol. 25, No. 2, pp. 796-808, May 2010.

[16] A. Hasanzadeh, O. C. Onar, H. Mokhtari, and A. Khaligh, "A proportional-resonant controller-based wireless control strategy with a reduced number of sensors for parallel-operated UPSs," IEEE Trans. Power Del., Vol. 25, No. 1, pp. 468-478, Jan. 2010.

[17] I. Y. Chung, W. Liu, D. A. Cartes, E. G. Collins Jr., and S.-I. Moon, "Control methods of inverter interfaced distributed generators in a microgrid system," IEEE Trans. Ind. Appl., Vol. 46, No. 3, pp. 1078-1088, May 2010.

[18] S. Golestan, M. Joorabian, H. Rastegar, A. Roshan, A. and J. M. Guerrero, "Droop based control of parallel-connected single-phase inverters in D-Q rotating frame," in Proc. ICIT, pp. 1-6, 2009.

[19] M. C. Chandrokar, D. M. Divan, and B. Banerjee, "Control of distributed UPS systems," in Proc. PESC, pp. 197-204, 1994.

[20] J. M. Guerrero, J. C. Vasquez, J. Matas, L. G. de Vicuña, and M. Castilla, "Hierarchical control of droop-controlled $\mathrm{AC}$ and DC microgrids-a general approach toward standardization," IEEE Trans. Ind. Electron., Vol. 58, No. 1, pp. 158-172, Jan. 2011.

[21] X. Sun, Y.-S. Lee, and D. Xu, "Modeling, analysis, and implementation of parallel multi-inverter systems with instantaneous average-current-sharing scheme," IEEE Trans. Power Electron., Vol. 18, No. 3, pp. 844-856, May 2003.

[22] J. Kim, J. M. Guerrero, P. Rodriguez, R. Teodorescu, and K. Nam, "Mode adaptive droop control with virtual output impedances for an inverter-based flexible AC microgrid," IEEE Trans. Power Electron., Vol. 26, No. 3, pp. 689-701, Mar. 2011.

[23] P. Jain and S. J. Ranade, "Capacity discovery in customer-driven micro-grids," in Proc. NAPS, pp. 1-6, 2009.

[24] P. Jain, S. J. Ranade, and S. K. Srivastava, "Island identification in customer-driven micro-grids," in Proc. IEEE PES T\&D, pp. 1-7, 2010.

[25] Y. L. Xu and X. Liu, "Novel multiagent based Load restoration algorithm for microgrids," IEEE Trans. Smart Grid, Vol. 2, No. 1, pp. 152-161, Mar. 2011.

[26] Y. L. Xu, W. Zhang, W. Liu, and F. Ferrese, "Multiagent-based reinforcement learning for optimal reactive power dispatch," IEEE Trans. Syst., Man, Cybern.
C, Appl. Rev., Vol. 42, No. 6, pp. 1742-1751, Nov. 2012.

[27] Y. L. Xu, W. Liu, and J. Gong, "Stable multi-agent-based load shedding algorithm for power systems," IEEE Trans. Power Syst., Vol. 26, No. 4, pp. 2006-2014, Nov. 2011.

[28] J. M. Guerrero, J. C. Vasquez, J. Matas, M. Castilla, and L. G. de Vicuna, "Control strategy for flexible microgrid based on parallel line-interactive UPS systems," IEEE Trans. Ind. Electron., Vol. 56, No. 3, pp. 726-736, Mar. 2009.

[29] X. Hu and Q. Cheng, "Application of UPFC based on improved double-loop decoupling PI control in photovoltaic systems," in Proc. CDCIEM, pp. 153-157, 2012.

[30] K. de Brabandere, B. Bolsens, J. Van den Keybus, A. Woyte, J. Driesen, and R. Belmans, "A voltage and frequency droop control method for parallel inverters," IEEE Trans. Power Electron., Vol. 22, No. 4, pp. 1107-1115, Jul. 2007

[31] J. M. Guerrero, J. Matas, L. G. de Vicuna, M. Castilla, and J. Miret, "Decentralized control for parallel operation of distributed generation inverters using resistive output impedance," IEEE Trans. Ind. Electron., Vol. 54, No. 2, pp. 994-1004, Apr. 2007.

[32] Y. Li and Y. W. Li, "Power management of inverter interfaced autonomous microgrid based on virtual frequency-voltage frame," IEEE Trans. Smart Grid, Vol. 2, No. 1, pp. 30-40, Mar. 2011.

[33] K. Strunz, R. H. Fletcher, R. Campbell, and F. Gao, "Developing benchmark models for low-voltage distribution feeders," in Proc. IEEE Power Energy Soc. Gen. Meet., PES, pp. 1-3, 2009.

[34] T. L. Vandoorn, B. Meersman, J. D. M. de Kooning, and L. Vandevelde, "Analogy between conventional grid control and islanded microgrid control based on a global DC-Link voltage droop," IEEE Trans. Power Del., Vol. 27, No. 3, pp. 1405-1414, Jul. 2012

[35] J. M. Guerrero, L. G. de Vicuna, J. Matas, M. Castilla, and J. Miret, "Output impedance design of parallel-connected UPS inverters with wireless load-sharing control," IEEE Trans. Ind. Electron., Vol. 52, No. 4, pp. 1126-1135, Aug. 2005.

[36] X. T. Zhang, H. Zhang, J. M. Guerrero, and X. Ma "Reactive power compensation for parallel inverters without control interconnections in microgrid," in Proc. IECON, pp. 922-925, 2008.

[37] T. L. Vandoorn, J. D. M. de Kooning, B. Meersman, J. M. Guerrero, and L. Vandevelde, "Automatic power-sharing modification of $\mathrm{P} / \mathrm{V}$ droop controllers in low-voltage resistive microgrids," IEEE Trans. Power Del., Vol. 27, No. 4, pp. 2318-2325, Oct. 2012.

[38] F. Xiao, L. Wang, and Y. Jia, "Fast information sharing in networks of autonomous agents," in Proc. American Control Conference, pp. 4388-4393, 2008.

[39] R. Olfati-Saber, J. A. Fax, and R. M. Murray, "Consensus and cooperation in networked multi-agent systems," in Proc. IEEE, Vol. 95, No. 1, pp. 215-233, Jan. 2007.

[40] H. Liang, B. J. Choi, W. Zhuang, X. Shen, A. S. A. Awad, and A. Abdr, "Multiagent coordination in microgrids via wireless networks," IEEE Wireless Communications, Vol. 19, No. 3, pp. 14-22, Jun. 2012.

[41] A. E. Boubakri and L. A. C. Lopes, "Phase shift based equivalent virtual impedance loop for droop-controlled inverters," in Proc. EPE'14-ECCE, pp. 1-8, 2014.

[42] C. D. Li, S. K. Chaudhary, J. C. Vasquez, and J. M. Guerrero, "Power flow analysis algorithm for islanded LV 
microgrids including distributed generator units with droop control and virtual impedance loop," in Proc. APEC, pp. 3181-3185, 2014.

[43] IEEE Recommended Practices and Requirements for Harmonic Control in Electrical Power Systems, IEEE Std. 519-1992, pp. 1-112, 1993.

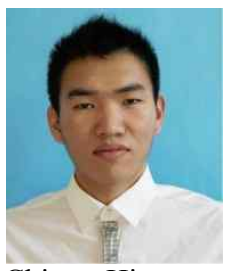

Zhongwen Li was born in Henan, China, in 1989. He received his B.S. degree in Control Science and Engineering from Zhengzhou University, Zhengzhou, China, in 2011. He is currently working toward his Ph.D. degree at the Shenyang Institute of Automation, Chinese Academy of Sciences, Shenyang, China. His current research interests include the control of inverter interfaced distributed generators and the optimization of microgrids.

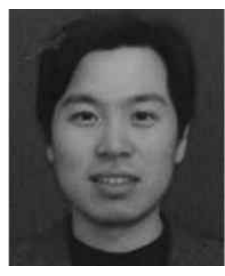

Chuanzhi Zang received his B.S. degree in Application Mathematics and his M.S. degree in Control Theory and Control Engineering from Northeastern University, Shenyang, China, in 1999 and 2002, respectively, and his Ph.D. degree in Mechatronic Engineering from the Graduate School of the Chinese Academy of Sciences, Shenyang, China, in 2006. He is an Associate Professor in the Shenyang Institute of Automation, Chinese Academy of Sciences, Shenyang, China. His current research interest include wireless sensor networks, control theory and smart grids.

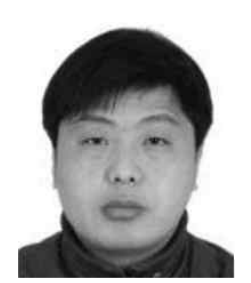

Peng Zeng received his B.S. degree in Computer Science from Shandong University, Shandong, China, in 1998, and his Ph.D. degree in Mechatronic Engineering from the Graduate School of the Chinese Academy of Sciences, Shenyang, China, in 2005. From 2005 to 2007, he was an Associate Professor with the Shenyang Institute of Automation, Chinese Academy of Sciences, Shenyang, China, where he was engaged in research on wireless sensor networks. He is presently a Professor with the Shenyang Institute of Automation, Chinese Academy of Sciences, Shenyang, China. His current research interests include wireless sensor networks for industrial automation, smart grids, and demand response.

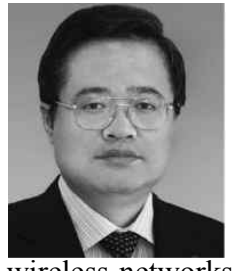

Haibin Yu received his B.S., and M.S. degrees in Automation Engineering and his Ph.D. degree in Control Theory and Control Engineering from Northeastern University, Shenyang, China, in 1984, 1987, and 1997, respectively. He has more than 25 years of research experience in fieldbus, industrial wireless networks, wireless sensor networks, network controlled systems, and network manufacturing. He is presently the President and a Professor of the Shenyang Institute of Automation (SIA), Chinese Academy of Sciences (CAS), Shenyang, China. His current research interests include basic and applied research in the areas of automation control systems, advanced manufacturing techniques and smart grids. Dr. Yu is a Senior Member of the Instrumentation, Systems, and Automation Society.

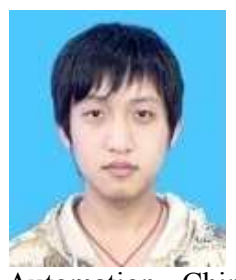

Hepeng $\mathbf{L i}$ received his B.S. degree in Applications Mathematics and his M.S. degree in Control Theory and Control Engineering from Northeastern University, Shenyang, China, in 2010 and 2012, respectively. $\mathrm{He}$ is presently a Research Assistant at the Shenyang Institute of Automation, Chinese Academy of Sciences, Shenyang, China. His current research interests include the control and optimization of microgrids.

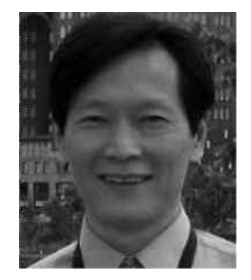

Shuhui Li received his B.S. and M.S. degrees in Electrical Engineering from Southwest Jiaotong University, Chengdu, China, in 1983 and 1988, respectively, and his Ph.D. degree in Electrical Engineering from Texas Tech University, Lubbock, TX, USA, in 1999. From 1988 to 1995 , he was with the School of Electrical Engineering at Southwest Jiaotong University, where his research interests included the modeling and simulation of large dynamic systems, dynamic process simulations of electrified railways, power electronics, power systems, and power system harmonics. From 1995 to 1999, he was engaged in research on wind power, artificial neural networks, and the application of massive parallel processing. He joined Texas A\&M University, Kingsville, TX, USA, as an Assistant Professor, in 1999, and then as an Associate Professor, in 2003. He worked with the Oak Ridge National Laboratory, Oak Ridge, TN, on simulation system development on supercomputers, from 2004 and 2006. He joined the University of Alabama, Tuscaloosa, AL, USA, as an Associate Professor, in 2006. His current research interests include renewable energy systems, smart grids, smart microgrids, power electronics, power systems, electric machines and drives, FACTS, distributed generation, and the application of artificial neural networks in energy systems. 